\title{
Anterior Scleral Thickness in Myopic Eyes and Its Association with Ocular Parameters
}

\author{
Mi Sun Sung Yong Sok Ji Hyun Sik Moon Hwan Heo Sang Woo Park \\ Department of Ophthalmology and Research Institute of Medical Sciences, Chonnam National University \\ Medical School and Hospital, Gwangju, South Korea
}

\section{Keywords}

Anterior scleral thickness - Myopia · Bruch's membrane opening · Anterior segment optical coherence tomography

\begin{abstract}
Purpose: This study was designed to determine whether the anterior scleral thickness is affected by axial elongation and to investigate the association between anterior scleral thickness and various ocular parameters in myopic eyes. Methods: This cross-sectional study included 79 healthy myopic participants. Anterior scleral thickness was measured in 8 meridians (superior-nasal, superior, superior-temporal, temporal [T], inferior-temporal, inferior [I], inferior-nasal, and nasal [N]) using anterior segment optical coherence tomography. Bruch's membrane opening (BMO) area, width of $\beta$-parapapillary atrophy with and without Bruch's membrane $\left(\mathrm{PPA}_{+\mathrm{BM}}\right.$ and $\left.\mathrm{PPA}_{-\mathrm{BM}}\right)$, and the global peripapillary and subfoveal choroidal thickness were additionally measured. Age- and sex-adjusted partial correlation analysis and linear regression analysis were performed to examine the effects of axial length and various ocular parameters on anterior scleral thickness in myopic eyes. Results: The mean age of the included participants was $27.03 \pm 2.70$ years. Overall, the anterior scleral thickness varied topographically according to the meridians and distance from the scleral spur. In the
\end{abstract}

partial correlation analysis controlled for the effect of age and sex, increasing axial length was related to anterior scleral thinning at several measurement points along the $T, I$, and $\mathrm{N}$ meridians. Among the several ocular parameters, multivariate linear regression analysis with age, sex, and axial length as covariates revealed that central corneal thickness, intraocular pressure, and $\mathrm{BMO}$ area were significantly associated with anterior scleral thickness. Conclusion: In conclusion, there was a close relationship between the anterior scleral thickness and several ocular parameters in myopic eyes. These features should be taken into consideration when managing myopia, and our results might have important implications for understanding the pathogenesis of scleral changes during axial elongation.

\footnotetext{
(c) 2020 The Author(s)

Published by S. Karger AG, Basel
}

\section{Introduction}

The sclera forms around $85 \%$ of the outer coat of the human eyeball, and its anterior boundary merges with the cornea at the limbus. The sclera is a structurally complex connective tissue, composed mainly of collagen fibrils in

Mi Sun Sung and Yong Sok Ji contributed equally to this work as first authors.
(C) 2020 The Author(s)

Published by S. Karger AG, Basel

This is an Open Access article licensed under the Creative Commons Attribution-NonCommercial-4.0 International License (CC BY-NC) (http://www.karger.com/Services/OpenAccessLicense), applicable to the online version of the article only. Usage and distribution for commercial purposes requires written permission.
Sang Woo Park

Department of Ophthalmology

Chonnam National University Medical School and Hospital

Dong-gu, Jebongro 42, Gwangju 61469 (South Korea) exo70@ naver.com 
a hydrated interfibrillar matrix of proteoglycans and glycoproteins [1]. Despite its bradytrophic nature and low cellular concentration, it has been shown that the human sclera undergoes extensive changes during axial elongation [2-6]. More importantly, animal studies have revealed that these scleral remodeling processes have an important role in the development of axial myopia. Increasing evidence suggests that the myopization process relies on a feedback mechanism that alters scleral remodeling to match the axial length of the eye to its optical system [2-7].

Until recently, morphologic changes in the posterior sclera have been a topic of significant interest in the field of myopia research [8-13]. There has been a large body of research regarding the changes of the posterior sclera in myopic eyes. It is generally accepted that axially elongated eyes show significant scleral thinning and tissue loss in the posterior pole area. However, little attention has been given to the anterior sclera, and there is limited information in this regard. Although several studies have investigated the anterior sclera in myopic eyes, the results are conflicting [5, 14-16]. Previous histomorphometric studies have demonstrated that anterior scleral thickness measured at the limbus and ora serrata was not significantly different in nonaxially elongated eyes and axially elongated eyes [5]. Buckhurst et al. [14] and Pekel et al. [15] also reported similar findings that axial length was not associated with the anterior scleral thickness using in vivo measurements of the sclera using optical coherence tomography (OCT), whereas more recently, Dhakal et al. [16] demonstrated significant thinning of the anterior sclera along the inferior meridian with increasing axial length.

Myopia is associated with an increased prevalence of glaucoma and maculopathy $[17,18]$. Given the increasing incidence of myopia, the anterior sclera is of particular interest since it is an important site for surgical procedures, such as trabeculectomy and vitrectomy, and future transscleral drug delivery. Additionally, understanding of the characteristics of the anterior sclera in myopic eyes may also help to better understand the underlying mechanism of various ocular diseases in myopic eyes. The goal of this study was to determine whether the anterior scleral thickness is affected by axial elongation. In addition, we hypothesized that there might be topographic variation in anterior scleral changes in myopic eyes according to their ocular features and investigated the associations between anterior scleral thickness and various ocular parameters.

\section{Methods}

\section{Participants}

This cross-sectional study was approved by the Institutional Review Board of Chonnam National University Hospital and followed the tenets of the Declaration of Helsinki. All participants provided written informed consent after a thorough explanation about the study.

Study participants were recruited from the Young Myopia Study of Chonnam National University Hospital. Detailed descriptions of our study participants and protocols have been published [19-21]. Briefly, the Young Myopia Study enrolled consecutive participants who visited the general eye clinic for medical checkup and met all of the inclusion criteria and none of the exclusion criteria. All participants underwent comprehensive ophthalmic examinations, including the measurement of best-corrected visual acuity, intraocular pressure (IOP) by using Goldmann applanation tonometry, manifest refraction, slit-lamp examination, anterior chamber angle examination by gonioscopy, optic nerve head $(\mathrm{ONH})$, and retinal nerve fiber layer (RNFL) examination using color stereoscopic disc photography and red-free RNFL fundus photography, and Swedish Interactive Threshold Algorithm standard 30-2 perimetry with a Humphrey Field Analyzer (Carl Zeiss Meditec Inc., Dublin, CA, USA). Axial length, central corneal thickness (CCT), and corneal curvature were measured using optical low-coherence reflectometry (Lenstar; Haag-Streit AG, Koeniz, Switzerland). A detailed medical history was also recorded for each participant.

The following inclusion criteria were used: (1) healthy individuals aged between 20 and 35 years, (2) a spherical equivalent refractive error between -12.0 and -0.5 diopters, (3) astigmatism within $\pm 2 \mathrm{D}$, (4) best-corrected visual acuity of $20 / 25$ or better, (5) IOP $\leq 21 \mathrm{~mm} \mathrm{Hg}$, (6) normal anterior chamber angles, (7) nonglaucomatous ONHs on stereoscopic photographs (an intact neuroretinal rim without peripapillary hemorrhage, thinning, or localized pallor), (8) absence of any RNFL abnormalities on red-free fundus photographs, and (9) normal visual field results in both eyes. Exclusion criteria were a family history of glaucoma in a firstdegree relative, history of intraocular or refractive surgery, pathologic myopia (patch chorioretinal atrophy, lacquer crack lesions, intrachoroidal cavitations, or choroidal neovascularization), other evidence of retinal pathology, opaque media, or poor-quality OCT images because of irregular tear film or poor cooperation. In cases where both the eyes of a participant met the inclusion criteria, only 1 eye was randomly selected for the study. The methods regarding participant enrollment were described by our previously published studies [19-21].

\section{Anterior Segment Imaging}

All anterior segment images were collected using the enhanced high-resolution scan mode of the Visante anterior segment-OCT (AS-OCT; Carl Zeiss Meditec, Dublin, CA, USA). The high-resolution images were generated from $512 \mathrm{~A}$-scans in $250 \mathrm{~ms}$. To acquire the scleral images of 8 different meridians (superior-nasal [SN], superior [S], superior-temporal, temporal [T], inferior-temporal [IT], inferior [I], inferior-nasal [IN], and nasal [N]), participants were asked to fixate in 8 directions external to the OCT instrument, and the upper or lower eyelid was gently extracted to expose the bulbar conjunctiva, taking care to avoid pressure on the globe. Radial scan sections perpendicular to the limbus were obtained in each of the 8 meridians. 
Fig. 1. Anterior segment optical coherence tomography image of the anterior sclera along the nasal meridian. Anterior scleral thickness measurements were taken at the SS and distances of 1-, 2-, 3-, 4-, 5-, and 6-mm points from the SS. SS, scleral spur.

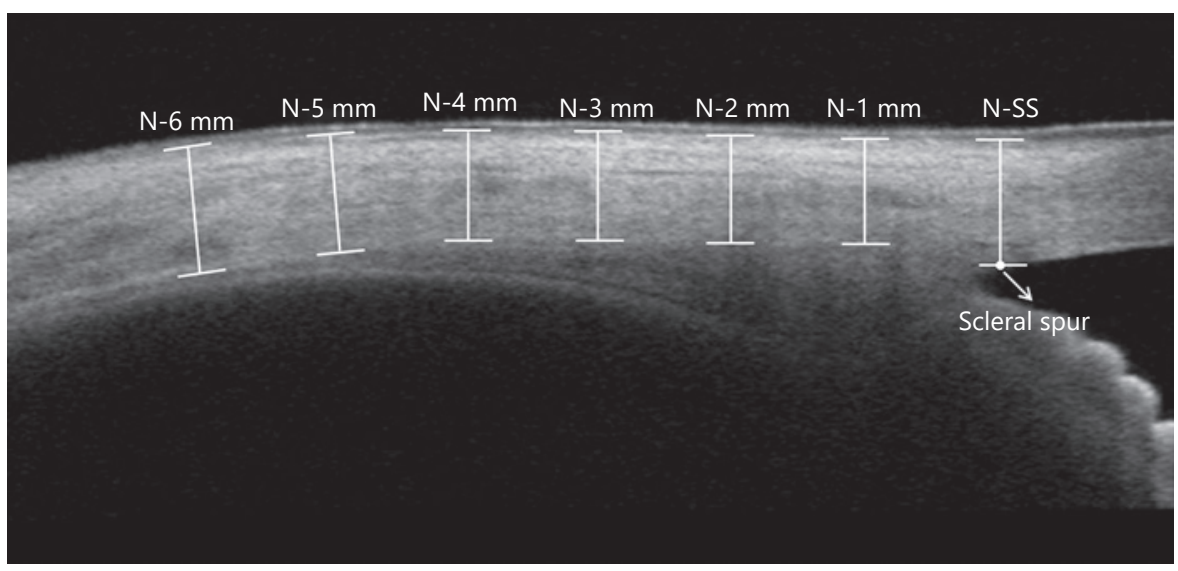

All scans were analyzed manually in random order by 2 independent examiners (M.S.S. and H.S.M.) in a blind manner. The outer scleral boundary was clearly identified by the line between the hyper-reflective scleral tissue and hypo-reflective episcleral vascular plexus, and the inner boundary was determined by the line between the hyper-reflective scleral tissue and hypo-reflective ciliary body tissue. The scleral thickness was measured by the distance from the outer scleral boundary to the inner scleral boundary. After determination of the apex of scleral spur (SS), where there is a slight protrusion of sclera into the anterior chamber and where the ciliary muscle fibers initially meet the sclera at the iridoscleral junction, the anterior scleral thickness at SS and at 6 discrete locations situated at 1,2, 3, 4, 5, and $6 \mathrm{~mm}$ from the SS was measured via manual calipers available in the OCT software (Fig. 1). The mean of the values obtained by the 2 examiners was used in the final analysis. The anterior scleral thickness measurement in this study was performed using a previously described method [14].

\section{Optic Nerve Head Measurements}

The ONH was examined using the spectral domain-OCT (Heidelberg Spectralis SD-OCT; Spectralis software version 6.9.4; Heidelberg Engineering $\mathrm{GmbH}$, Heidelberg, Germany). All OCT scans were performed by an experienced technician (M.Y.H.). Magnification error was corrected using the formula provided by the manufacturer, based on the keratometry results and focus setting during image acquisition. If OCT scan had inadequate image quality (typically truncated B-scans or quality score $<30$ ), that eye was excluded from analyses.

Bruch's membrane opening (BMO) area was measured using the Glaucoma Module Premium Edition software. Our strategy for the determination of BMO points and the measurement of BMO area has been described previously $[19,21]$. The ONH was additionally scanned using the enhanced depth imaging mode for the other $\mathrm{ONH}$ measurements. The $\mathrm{ONH}$ was scanned by centering a $15^{\circ} \times 10^{\circ}$ rectangular scan on the ONH. Each OCT volume consisted of 49 serial horizontal B-scans (4.5-mm-long lines; 50 images averaged) spaced at approximately $63-\mu \mathrm{m}$ intervals. Infrared fundus images were acquired simultaneously by using a confocal scanning laser ophthalmoscope. Three sections that passed through the $\mathrm{ONH}$ in the center, mid-superior, and mid-inferior regions were selected, and all the study parameters were measured
Table 1. Demographics of the 79 myopic subjects

\begin{tabular}{lc}
\hline Variables & Description \\
\hline Age, years & $27.03 \pm 2.70(24-35)$ \\
Male, $n(\%)$ & $51(64.56)$ \\
SE refractive error, D & $-5.06 \pm 2.80(-10.75$ to -0.50$)$ \\
Axial length, mm & $25.91 \pm 1.36(22.84-28.63)$ \\
CCT, $\mu$ m & $549.58 \pm 33.70(447-611)$ \\
Corneal curvature, D & $42.89 \pm 1.26(40.12-45.68)$ \\
IOP, mm Hg & $13.82 \pm 2.10(10-20)$ \\
BMO area, mm ${ }^{2}$ & $2.55 \pm 0.61(1.53-4.38)$ \\
PPA + BM width, $\mu \mathrm{m}$ & $195.10 \pm 189.23(0-882)$ \\
PPA_BM width, $\mu \mathrm{m}$ & $267.79 \pm 205.54(0-757)$ \\
Global peripapillary CT, $\mu \mathrm{m}$ & $154.47 \pm 44.08(73-270)$ \\
Subfoveal CT, $\mu \mathrm{m}$ & $252.52 \pm 75.87(103-393)$ \\
\end{tabular}

Continuous variables are shown as mean \pm standard deviation (range). SE, spherical equivalent; D, diopters; CCT, central corneal thickness; IOP, intraocular pressure; BMO, Bruch's membrane opening; $\mathrm{PPA}_{+\mathrm{BM}}, \beta$-parapapillary atrophy with Bruch's membrane; PPA-BM, $\beta$-parapapillary atrophy without Bruch's membrane, CT, choroidal thickness.

in each of these frames by 2 independent examiners (M.S.S. and H.H.), in a masked fashion.

Temporal $\beta$-zone PPA margin, $\mathrm{BMO}$, and disc margin were defined using infrared fundus images. $\beta$-PPA width was measured as the distance between the beginning of the retinal pigment epithelium (RPE) (i.e., temporal $\beta$-PPA margin) and temporal disc margin on each horizontal B-scan image. On the basis of the location of $\mathrm{BM}$ termination, the $\beta$-PPA was further divided into $\mathrm{PPA}_{+\mathrm{BM}}$ and $\mathrm{PPA}_{-\mathrm{BM}}\left(\gamma\right.$-zone PPA); $\mathrm{PPA}_{+\mathrm{BM}}$ width was defined as the distance from the beginning of the RPE to BMO, and PPA-BM was defined as the distance from the temporal disc margin to the beginning of BM. The measurement was performed using a previously described method [19-22].

The measurement was performed using a built-in caliper tool of the intrinsic OCT viewer, and the average data of 3 horizontal $\mathrm{B}$-scan images (center, mid-superior, and mid-inferior) were cal- 
Table 2. Anterior scleral thickness at each meridian from SS to the 6-mm periphery with 1-mm intervals in myopic eyes

\begin{tabular}{|c|c|c|c|c|c|c|c|c|}
\hline Meridians & SS & $1 \mathrm{~mm}$ & $2 \mathrm{~mm}$ & $3 \mathrm{~mm}$ & $4 \mathrm{~mm}$ & $5 \mathrm{~mm}$ & $6 \mathrm{~mm}$ & Average* \\
\hline $\mathrm{SN}$ & $708.9 \pm 65.0$ & $632.2 \pm 57.4$ & $647.1 \pm 55.2$ & $663.3 \pm 57.3$ & $685.9 \pm 60.1$ & $709.9 \pm 61.9$ & $743.9 \pm 69.6$ & $680.4 \pm 56.8$ \\
\hline S & $757.2 \pm 66.9$ & $681.2 \pm 61.8$ & $696.8 \pm 58.7$ & $714.0 \pm 61.5$ & $736.6 \pm 68.7$ & $764.0 \pm 71.3$ & $808.8 \pm 75.1$ & $733.5 \pm 62.5$ \\
\hline ST & $763.7 \pm 79.2$ & $685.8 \pm 68.3$ & $702.4 \pm 69.1$ & $723.3 \pm 73.9$ & $741.9 \pm 78.4$ & $765.6 \pm 81.2$ & $801.4 \pm 87.2$ & $736.7 \pm 74.1$ \\
\hline $\mathrm{T}$ & $840.6 \pm 67.9$ & $765.9 \pm 77.4$ & $773.2 \pm 63.8$ & $789.9 \pm 60.3$ & $802.7 \pm 67.7$ & $820.0 \pm 75.5$ & $864.1 \pm 70.5$ & $802.6 \pm 58.8$ \\
\hline IT & $827.1 \pm 61.2$ & $766.1 \pm 54.5$ & $771.0 \pm 52.3$ & $783.0 \pm 58.6$ & $802.9 \pm 67.8$ & $819.2 \pm 80.8$ & $858.1 \pm 78.6$ & $800.0 \pm 58.1$ \\
\hline I & $839.0 \pm 53.4$ & $777.8 \pm 54.1$ & $789.0 \pm 48.0$ & $801.3 \pm 63.6$ & $823.6 \pm 67.3$ & $852.6 \pm 82.8$ & $902.9 \pm 78.2$ & $824.3 \pm 55.0$ \\
\hline IN & $797.7 \pm 70.1$ & $742.0 \pm 70.7$ & $750.6 \pm 65.3$ & $764.3 \pm 64.3$ & $770.8 \pm 71.9$ & $786.2 \pm 84.0$ & $821.1 \pm 76.5$ & $772.5 \pm 65.7$ \\
\hline $\mathrm{N}$ & $760.9 \pm 63.0$ & $676.2 \pm 62.4$ & $702.9 \pm 59.7$ & $734.1 \pm 63.8$ & $763.7 \pm 67.3$ & $797.2 \pm 69.4$ & $843.3 \pm 73.2$ & $752.9 \pm 61.1$ \\
\hline Average thickness & $786.8 \pm 79.2$ & $715.9 \pm 80.9$ & $729.1 \pm 74.9$ & $746.6 \pm 76.4$ & $766.0 \pm 80.2$ & $789.3 \pm 86.2$ & $830.3 \pm 88.2$ & $762.8 \pm 75.4$ \\
\hline
\end{tabular}

Values are shown as mean \pm standard deviation $(\mu \mathrm{m})$. SS, scleral spur; SN, superior-nasal; S, superior; ST, superior-temporal; T, temporal; IT, inferior-temporal; I, inferior; IN, inferior-nasal; N, nasal. * Averaged anterior scleral thickness of each meridian from 1- to 6-mm points from the limbus.

culated and used in this study. The mean of the values obtained by the 2 examiners was used in the final analysis.

\section{Choroid Thickness Measurement}

We obtained $360^{\circ}$ circular RNFL measurements centered on the $\mathrm{BMO}$ center. Among the 3 circular scans along the peripapillary circles (diameters of 3.5, 4.1, and $4.7 \mathrm{~mm}$ ), the $4.1-\mathrm{mm}$ diameter scans were analyzed to minimize the interference of a large PPA on the OCT scan path. For the measurement of peripapillary choroidal thickness (CT), the upper and lower segmentation lines of the circular scan were manually delineated. The lines were adjusted to align with the inner scleral wall and the posterior border of the RPE to define the outer and inner boundaries of the choroid, respectively. Global peripapillary CT was automatically computed using the RNFL thickness measurement algorithms. Subfoveal CT was measured from the enhanced depth imaging scan running through the fovea. The vertical distance from the hyper-scattering outer border of the RPE to the inner border of the sclera at the fovea was defined as subfoveal choroidal thickness. Images in which the RPE and chorioscleral interface could not be clearly identified were excluded from the analysis. The average of data from the 2 independent examiners (M.S.S. and H.H.) was used in this study. The measurement was performed using a previously described method [19-22].

\section{Statistical Analysis}

All statistical analyses were performed using SPSS software version 21.0 (Chicago, IL, USA). Agreement on PPA + BM width, PPA $_{-B M}$ width, peripapillary and subfoveal $\mathrm{CT}$, and anterior scleral thickness between the 2 observers was assessed using the Bland-Altman method, which plots the means against differences [23]. The limits of agreement were defined as the mean differences of 2 measurements \pm 1.96 standard deviation of the difference. The normality of distribution was verified using the Shapiro-Wilk normality test. All data were normally distributed, except for the $\mathrm{PPA}_{+\mathrm{BM}}$ and $\mathrm{PPA}_{-\mathrm{BM}}$ width. The partial correlation analysis was used to investigate the relationship between the anterior scleral thickness and axial length adjusted for age and sex. Linear regressions were conducted to assess the relationship between the anterior scleral thickness at each measurement points and various ocular parameters after including age, sex, and axial length as covariates. Coefficients with $95 \%$ confidence intervals were presented. A $p$ value of $<0.05$ was considered statistically significant.

\section{Results}

Seventy-nine myopic eyes of 79 participants were included in the analysis. Demographic and ocular data for the participants are summarized in Table 1 . The mean age of the participants was $27.03 \pm 2.70$ years, and 51 participants were men. The mean spherical equivalent refractive error and axial length were $-5.06 \pm 2.80 \mathrm{D}$ and $25.91 \pm 1.36 \mathrm{~mm}$, respectively. The interobserver agreement in the measurements of the PPA $+B M$ width, PPA_BM width, peripapillary and subfoveal CT, and average anterior scleral thickness from each meridians for all subjects is represented in Bland-Altman plots, showing no systematic differences in measurements (see online suppl. Fig. 1, 2; for all online suppl. material, see www.karger.com/doi/10.1159/000512396).

Table 2 and Figure 2 show the profile of anterior scleral thickness at each meridian in myopic eyes. Overall, the anterior scleral thickness varied topographically according to the meridians and distance from SS. The anterior scleral thickness was thinnest at the $1-\mathrm{mm}$ points from SS and then shows a general trend for gradual increase toward the $6-\mathrm{mm}$ points from SS. Figure $2 \mathrm{a}$ and $\mathrm{b}$ illustrates the average anterior scleral thickness of 6 measurement points (1, $2,3,4,5$, and $6 \mathrm{~mm}$ from SS) and anterior scleral thickness of SS points, respectively, at each meridian. The anterior sclera was thinnest at the SN meridian, whereas the T, IT, and I meridians showed generally thicker sclera. 
Table 3. Age- and sex-adjusted partial correlation analysis between anterior sclera thickness and axial length in myopic eyes

\begin{tabular}{|c|c|c|c|c|c|c|c|}
\hline \multirow[t]{2}{*}{ Meridians } & \multicolumn{7}{|c|}{ Associations with axial length } \\
\hline & SS & $1 \mathrm{~mm}$ & $2 \mathrm{~mm}$ & $3 \mathrm{~mm}$ & $4 \mathrm{~mm}$ & $5 \mathrm{~mm}$ & $6 \mathrm{~mm}$ \\
\hline S & $0.033(0.773)$ & $0.072(0.534)$ & $0.059(0.607)$ & $0.033(0.772)$ & $0.005(0.966)$ & $0.014(0.901)$ & $-0.003(0.977)$ \\
\hline ST & $-0.121(0.287)$ & $-0.068(0.551)$ & $-0.138(0.227)$ & $-0.160(0.159)$ & $-0.219(0.052)$ & $-0.215(0.057)$ & $-0.203(0.072)$ \\
\hline $\mathrm{T}$ & $-0.261(0.020)$ & $-0.254(0.024)$ & $-0.279(0.013)$ & $-0.271(0.016)$ & $-0.204(0.071)$ & $-0.128(0.262)$ & $-0.197(0.082)$ \\
\hline IN & $-0.150(0.188)$ & $-0.120(0.294)$ & $-0.087(0.448)$ & $-0.122(0.283)$ & $-0.117(0.306)$ & $-0.138(0.227)$ & $-0.189(0.095)$ \\
\hline $\mathrm{N}$ & $-0.094(0.409)$ & $-0.011(0.925)$ & $-0.153(0.179)$ & $-0.207(0.067)$ & $-0.267(0.017)$ & $-0.261(0.020)$ & $-0.273(0.015)$ \\
\hline
\end{tabular}

Values are shown as the correlation coefficients with $p$ values in parenthesis. Statistically significant values are bold. SS, scleral spur; SN, superior-nasal; S, superior; ST, superior-temporal; T, temporal; IT, inferior-temporal; I, inferior; IN, inferior-nasal; N, nasal.

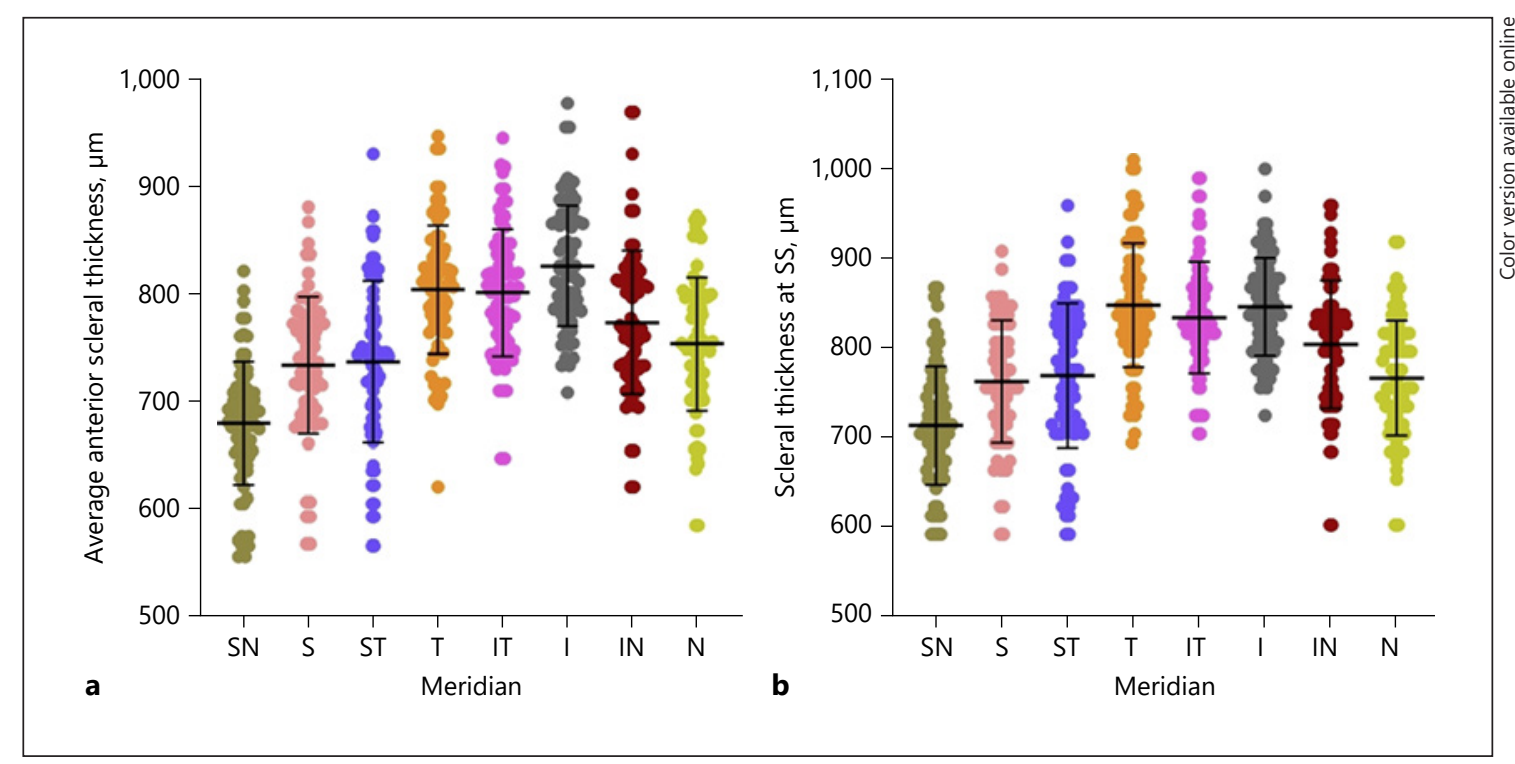

Fig. 2. Anterior scleral thickness at 8 different meridians. a Average anterior scleral thickness of 6 measurement points $(1,2,3,4,5$, and $6 \mathrm{~mm}$ from SS) at the 8 different meridians. b Anterior scleral thickness of SS points at the 8 different meridians. SS, scleral spur; SN, superior-nasal; S, superior; ST, superior-temporal; T, temporal; IT, inferior-temporal; I, inferior; IN, inferior-nasal; N, nasal.

In the partial correlation analysis controlled for the effect of age and sex, we found that increasing axial length was related to anterior scleral thinning at several measurement points. Anterior scleral thickness at the SS, 1-, $2-$, and $3-\mathrm{mm}$ points along the $\mathrm{T}$ and I meridians and at the 4-, 5-, and 6-mm points along the $\mathrm{N}$ meridians decreased significantly with increasing axial length (Table 3).

Among the ocular parameters, multivariate linear regression analysis with age, sex, and axial length as covari- ates revealed that CCT, IOP, and the BMO area were significantly associated with anterior scleral thickness. Particularly, a thinner overall anterior scleral thickness was associated with a large BMO area. However, the impact of ocular parameters on anterior scleral thickness varied across the meridians and the distance from the SS. No significant effect was found for the ocular parameters at the S, SN, and IN meridians (Table 4). Corneal curvature, $\mathrm{PPA}_{+\mathrm{BM}}$ width, PPA ${ }_{-\mathrm{BM}}$ width, and global peripapillary and subfoveal CT did not show any significant associa- 
Table 4. Age-, sex-, and axial length-adjusted linear regression results describing the relationships between the ocular variables and the anterior scleral thickness in myopic eyes

\begin{tabular}{|c|c|c|c|c|}
\hline Variables & $\begin{array}{l}\text { Location of } \\
\text { anterior sclera }\end{array}$ & B (95\% CI) & $p$ value & $R^{2 *}$ \\
\hline \multirow[t]{8}{*}{ CCT } & $\mathrm{I}-1 \mathrm{~mm}$ & $0.00033(0.000-0.00066)$ & 0.049 & 0.235 \\
\hline & $\mathrm{I}-2 \mathrm{~mm}$ & $0.00043(0.000-0.00073)$ & 0.006 & 0.194 \\
\hline & $\mathrm{I}-3 \mathrm{~mm}$ & $0.00045(0.000-0.00086)$ & 0.029 & 0.163 \\
\hline & $\mathrm{N}-1 \mathrm{~mm}$ & $0.00065(0.000-0.00105)$ & 0.002 & 0.159 \\
\hline & $\mathrm{N}-2 \mathrm{~mm}$ & $0.00064(0.000-0.001)$ & 0.001 & 0.213 \\
\hline & $\mathrm{N}-3 \mathrm{~mm}$ & $0.00057(0.000-0.00096)$ & 0.005 & 0.206 \\
\hline & $\mathrm{N}-4 \mathrm{~mm}$ & $0.00052(0.000-0.00094)$ & 0.014 & 0.212 \\
\hline & N-5 mm & $0.00051(0.000-0.00093)$ & 0.018 & 0.234 \\
\hline \multirow[t]{3}{*}{ IOP } & T-SS & $-0.014(-0.020$ to -0.008$)$ & $<0.001$ & 0.375 \\
\hline & $\mathrm{T}-1 \mathrm{~mm}$ & $-0.014(-0.021$ to -0.006$)$ & 0.001 & 0.253 \\
\hline & $\mathrm{T}-2 \mathrm{~mm}$ & $-0.010(-0.017$ to -0.004$)$ & 0.002 & 0.229 \\
\hline \multirow[t]{24}{*}{$\mathrm{BMO}$ area } & $\mathrm{ST}-1 \mathrm{~mm}$ & $-0.027(-0.053$ to -0.00029$)$ & 0.048 & 0.071 \\
\hline & $\mathrm{ST}-2 \mathrm{~mm}$ & $-0.030(-0.056$ to -0.004$)$ & 0.026 & 0.107 \\
\hline & $\mathrm{ST}-3 \mathrm{~mm}$ & $-0.030(-0.058$ to -0.002$)$ & 0.034 & 0.115 \\
\hline & $\mathrm{ST}-4 \mathrm{~mm}$ & $-0.031(-0.060$ to -0.002$)$ & 0.035 & 0.145 \\
\hline & ST-5 mm & $-0.032(-0.062$ to -0.002$)$ & 0.034 & 0.150 \\
\hline & T-SS & $-0.027(-0.050$ to -0.003$)$ & 0.028 & 0.244 \\
\hline & $\mathrm{T}-1 \mathrm{~mm}$ & $-0.035(-0.063$ to -0.007$)$ & 0.015 & 0.193 \\
\hline & $\mathrm{T}-2 \mathrm{~mm}$ & $-0.030(-0.053$ to -0.007$)$ & 0.011 & 0.199 \\
\hline & $\mathrm{T}-3 \mathrm{~mm}$ & $-0.027(-0.049$ to -0.005$)$ & 0.015 & 0.187 \\
\hline & $\mathrm{T}-4 \mathrm{~mm}$ & $-0.029(-0.054$ to -0.004$)$ & 0.023 & 0.138 \\
\hline & $\mathrm{T}-5 \mathrm{~mm}$ & $-0.029(-0.058$ to -0.001$)$ & 0.049 & 0.094 \\
\hline & $\mathrm{T}-6 \mathrm{~mm}$ & $-0.028(-0.054$ to -0.002$)$ & 0.037 & 0.134 \\
\hline & IT-SS & $-0.026(-0.049$ to -0.002$)$ & 0.032 & 0.100 \\
\hline & IT-1 mm & $-0.030(-0.050$ to -0.009$)$ & 0.005 & 0.126 \\
\hline & IT-2 mm & $-0.024(-0.044$ to -0.004$)$ & 0.020 & 0.099 \\
\hline & I-SS & $-0.023(-0.042$ to -0.004$)$ & 0.016 & 0.231 \\
\hline & $\mathrm{I}-1 \mathrm{~mm}$ & $-0.027(-0.045$ to -0.009$)$ & 0.005 & 0.277 \\
\hline & N-SS & $-0.024(-0.048$ to -0.001$)$ & 0.044 & 0.110 \\
\hline & $\mathrm{N}-1 \mathrm{~mm}$ & $-0.030(-0.053$ to -0.006$)$ & 0.014 & 0.115 \\
\hline & $\mathrm{N}-2 \mathrm{~mm}$ & $-0.029(-0.051$ to -0.007$)$ & 0.010 & 0.165 \\
\hline & $\mathrm{N}-3 \mathrm{~mm}$ & $-0.034(-0.056$ to -0.011$)$ & 0.004 & 0.210 \\
\hline & $\mathrm{N}-4 \mathrm{~mm}$ & $-0.033(-0.057$ to -0.009$)$ & 0.007 & 0.226 \\
\hline & $\mathrm{N}-5 \mathrm{~mm}$ & $-0.028(-0.052$ to -0.003$)$ & 0.028 & 0.226 \\
\hline & $\mathrm{N}-6 \mathrm{~mm}$ & $-0.028(-0.053$ to -0.002$)$ & 0.036 & 0.226 \\
\hline
\end{tabular}

Multivariable models are adjusted for age, sex, and axial length. Only statistically significant relationships are presented in this table. B, unstandardized coefficients; CI, confidence interval; CCT, central corneal thickness; IOP, intraocular pressure; SS, scleral spur; ST, superior-temporal; T, temporal; IT, inferior-temporal; I, inferior; IN, inferior-nasal; N, nasal; BMO, Bruch's membrane opening. * Adjusted coefficients of determination from the multivariate linear regression models including age, sex, axial length, and the each variables.

tions with anterior scleral thickness at any measurement point. Figure 3 demonstrates the schematic illustration of the significant associations between the ocular parameters and anterior scleral thickness. Colored dots indicate the measurement points that showed significant relationship with each parameter.

\section{Discussion}

Our study reports the distributions of anterior scleral thickness in otherwise healthy myopic eyes and its association with several ocular parameters. We observed similar trends in the distribution of anterior scleral thickness as reported in previous studies $[14,16]$, and in addition, we 
Fig. 3. Schematic illustration of the significant associations between ocular parameters and anterior scleral thickness. Colored dots indicate the measurement points that showed a significant relationship with each parameter. SS, scleral spur; SN, superiornasal; S, superior; ST, superior-temporal; T, temporal; IT, inferior-temporal; I, inferior; IN, inferior-nasal; $\mathrm{N}$, nasal; CCT, central corneal thickness; IOP, intraocular pressure; BMO, Bruch's membrane opening.

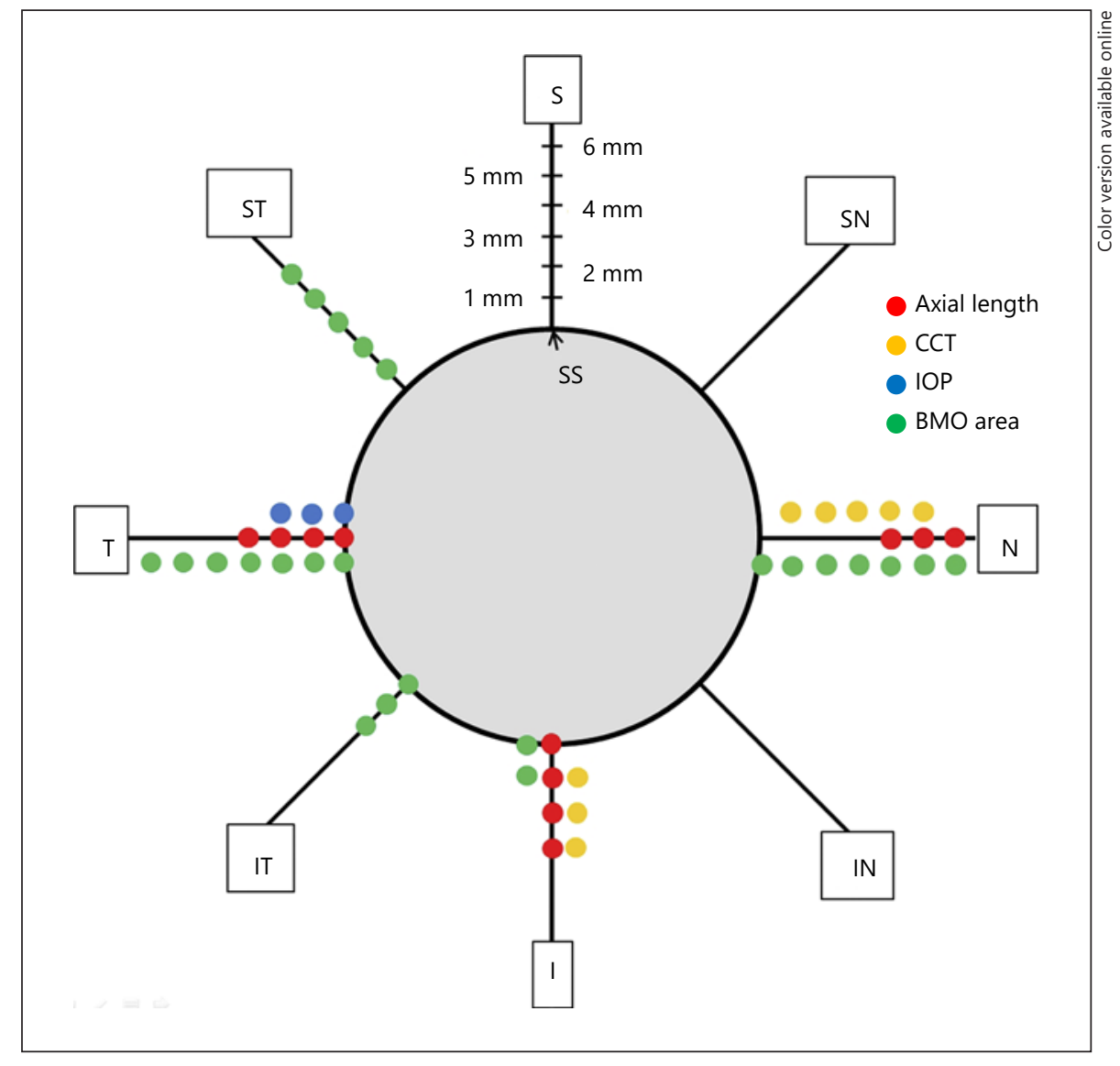

also found that anterior scleral thicknesses at several measurement points were influenced significantly by axial length, CCT, IOP, and BMO area in myopic eyes. Of note, these associations differed and varied across the measurement locations. To the best of our knowledge, this study, performed with a myopic population, is the first thorough analysis of the association between anterior scleral thickness and axial length and various ocular parameters.

Our study shows that the anterior sclera was thickest at the I meridian, followed by the IT, T, and IN meridians and thinnest at the SN meridian. The sclera was relatively thick near the limbus (SS), thinned at the $1-\mathrm{mm}$ points from the SS, and showed a gradual increase in thickness toward the 6-mm point from the SS. Although a direct comparison of our results with those of other reported studies is limited owing to differences in ethnicity, age, and degree of myopia of study population, the topographic variations found in this study are generally in line with the previous findings $[14,16,24,25]$.

Several studies have reported the characteristics of anterior sclera in normal eyes. Table 5 summarizes previous studies that reported determinants of anterior scleral thickness in healthy eyes [5, 14-16, 24-26]. Based on their findings, older and male participants exhibit thicker anterior scleral thickness, and the CCT and anterior chamber depth were positively associated with anterior scleral thickness. Additionally, Read et al. [26] demonstrated that anterior scleral thickness undergoes significant changes over a 24 -h period.

However, regarding the associations with axial length, the results have been conflicting. Most studies did not find a significant relationship between anterior scleral thickness and axial length $[5,14,15,25]$. The study by Buckhurst et al. [14] that had a similar study design and used the same OCT instruments as ours, also revealed that the axial length did not correlate with anterior scleral thickness at any meridian, whereas the present study observed statistically significant relationships with the axial length at $\mathrm{T}, \mathrm{I}$, and $\mathrm{N}$ meridians, albeit with borderline significance at several measurement points. The discrepancy in findings may be related to the confounding effects of age and sex; specifically, the previous studies did not consider the effects of age and sex when evaluating these relationships. 
Table 5. Factors associated with anterior scleral thickness reported in the previous studies

\begin{tabular}{|c|c|c|c|c|c|c|}
\hline Study & Years & Measurements & $N$ & Associated factors & Findings & Comments \\
\hline Vurgese et al. [5] & 2012 & $\begin{array}{l}\text { Histomorphometric } \\
\text { study }\end{array}$ & 238 & $\begin{array}{l}\text { Posterior scleral } \\
\text { thickness }\end{array}$ & $\begin{array}{l}\text { Anterior scleral thickness measured at ora } \\
\text { serrata was significantly associated with } \\
\text { posterior scleral thickness }\end{array}$ & $\begin{array}{l}\text { No associations with } \\
\text { axial length }\end{array}$ \\
\hline Pekel et al. [15] & 2015 & AS-OCT (Spectralis) & 62 & - & - & $\begin{array}{l}\text { No associations with } \\
\text { axial length }\end{array}$ \\
\hline Buckhurst et al. [14] & 2015 & AS-OCT (Visante) & 74 & Sex & Men showed greater anterior scleral thickness & $\begin{array}{l}\text { No associations with } \\
\text { axial length and } \\
\text { ethnicity }\end{array}$ \\
\hline Read et al. [25] & 2016 & AS-OCT (Spectralis) & 111 & $\begin{array}{l}\text { Age, sex, CCT, } \\
\text { ACD }\end{array}$ & $\begin{array}{l}\text { Older and male subjects exhibit greater anterior } \\
\text { scleral thickness. CCT and ACD were positively } \\
\text { associated with anterior scleral thickness }\end{array}$ & $\begin{array}{l}\text { No associations with } \\
\text { axial length }\end{array}$ \\
\hline Dhakal et al. [16] & 2020 & SS-OCT (topcon) & 95 & Axial length & $\begin{array}{l}\text { Anterior scleral thickness at inferior meridian } \\
\text { decreased with the increasing degree of myopia }\end{array}$ & $\begin{array}{l}\text { Associated with axial } \\
\text { length }\end{array}$ \\
\hline
\end{tabular}

AS-OCT, anterior segment-optical coherence tomography; SS-OCT, swept source-optical coherence tomography; CCT, central corneal thickness; ACD, anterior chamber depth.

The results of our investigation are somewhat consistent with the findings obtained in a recent study on an Indian myopic population, in which anterior scleral thickness at the SS and 1-, 2-, and 3-mm locations along the inferior meridian decreased significantly with increasing axial length [16]. In addition, we found statistically significant relationships with axial length along the temporal and nasal meridians. Dhakal et al. [16] speculated that disproportional anterior scleral thinning might be due to asymmetrical globe expansion (inferior more than superior) during axial elongation. However, we should note the lack of geographic continuity in the relationship with the axial length; specifically, no significant associations were found along the IT and IN meridians in this study. Furthermore, there were no statistically significant relationships with the axial length beyond the 3-mm points from the SS along the I and T meridians. Our results might indicate that the anterior scleral thinning observed in myopic eyes is not solely dependent on the pressure-induced passive ocular expansion that occurred during the axial elongation, suggesting the complexities of scleral biomechanics. There might be other factors affecting the anterior scleral thinning.

Zhang et al. [27] previously demonstrated that BMO has the tendency to stay stable until the axial length of $26.0 \mathrm{~mm}$, and beyond that limit, the BMO enlarged. They speculated that the process of myopic axial elongation leads to an increased strain within BM and subsequently causes enlargement of the physiological hole, BMO. However, we have observed that the BMO area varied among highly myopic eyes having similar axial lengths in the recent study [21]. Eyes with enlarged BMO exhibited characteristic morphologic features of peripapillary choroidal thinning and the deformation of lamina cribrosa [21]. We hypothesized that eyes with large BMO might have undergone higher mechanical strain on the posterior pole area during axial elongation. Interestingly, we found that BMO enlargement also accompanies overall anterior scleral thinning. Taken together, the BMO area might be a relative representation of individual biomechanical properties of the sclera and the enlarged BMO might imply biomechanically weaker sclera that tends to stretch more easily.

Our results showed that the anterior scleral thickness was influenced significantly by CCT and IOP. Although it is unclear whether the occurrence of these significant relationships is a coincidental finding or whether there is a causal relationship, it is reasonable to assume that CCT and IOP might play a certain role in anterior scleral thinning during myopization because the anterior sclera is continuous with the cornea and adjacent to the anterior chamber. Specifically, the anterior sclera transitions an- 
teriorly to become cornea at the limbus, and both anterior sclera and cornea constitute the anterior segment of the eyeball. Therefore, we thought that cornea and anterior sclera might share some characteristic biomechanical property, showing significant relationship in this study. In addition, since the IOP is the fluid pressure of anterior eye segment, IOP-induced scleral deformations could affect the thinning of adjacent tissue, anterior sclera, in myopic eyes. Whether these findings can be generalized to other ethnic population and other refractive error groups (emmetropes or hyperopes) remains to be determined.

It was interesting that anterior scleral thickness at the $\mathrm{S}, \mathrm{SN}$, and IN meridians was not related to any of the ocular parameters in this study, and the associations between anterior scleral thickness and several ocular parameters were marked at the $\mathrm{T}, \mathrm{I}$, and $\mathrm{N}$ meridians, which are the insertion meridians of the lateral rectus, inferior rectus, and medial rectus muscles, respectively. It has been suggested that eye movements might partly contribute to axial elongation in myopia. Based on several previous studies, the change in axial length might be due to mechanical forces generated by the ciliary muscles or extraocular muscles [28-30]. Although estimating their effects on globe geometry might be complex, since the extraocular muscles forces are not acting on the axial direction, we speculated that continuous mechanical tension by extraocular muscles might weaken the anterior sclera at the $\mathrm{T}, \mathrm{I}$, and $\mathrm{N}$ meridians. The connective tissue weakness and additional mechanical factors related to the extraocular muscles would explain why these meridians are especially susceptible for thinning by several ocular parameters. Regarding the absence of any significant relationships along the $\mathrm{S}$ meridians where the superior rectus muscles insert, it might be because the insertion of the superior rectus muscle is relatively far from the limbus compared with other rectus muscles [31]. Anterior sclera along the superior meridian could be less affected by rectus muscles. Future studies might be needed to account for regional variations in anterior scleral thickness and its association with ocular parameters.

There are several possible limitations to the present study. First, this study was cross-sectional, and therefore, we could not detect the temporal relationship between anterior scleral thickness and several ocular parameters. Longitudinal studies might be needed to further clarify the relationships found in this study. Second, one may argue regarding the accuracy of anterior scleral thickness using Visante AS-OCT. However, using the enhanced high-resolution scan mode, we could clearly identify the anterior scleral boundary in the study participants, and we strictly excluded the scan images with inadequate quality. Third, this study included only the Korean population; therefore, it is not clear whether the results of our study can be generally transferred onto other ethnic populations. Fourth, small sample sizes have certain limitations. Nevertheless, given the clinical importance of the anterior sclera for various surgical procedures and future therapeutics, our findings may have clinical relevance. Furthermore, our results have important implications for understanding the pathogenesis of scleral changes during axial elongation.

In conclusion, there was a close relationship between anterior scleral thickness and several ocular parameters in myopia. Future studies are needed to elucidate the cause of characteristic scleral thinning, and they may be potential avenues for determining the pathogenesis of various ocular disease in myopic eyes.

\section{Statement of Ethics}

This cross-sectional study was approved by the Institutional Review Board of Chonnam National University Hospital and followed the tenets of the Declaration of Helsinki (CNUH-2018-296). All participants provided written informed consent after a thorough explanation about the study.

\section{Conflict of Interest Statement}

The authors have no conflicting interests to disclose regarding any material discussed in this article.

\section{Funding Sources}

This research was supported by the Bio \& Medical Technology Development Program of the NRF funded by the Korean government (MSIP-2017M3A9E8023019), by a grant of Patient-Centered Clinical Research Coordinating Center funded by the Ministry of Health and Welfare, Republic of Korea (HI19C0481, HC19C0276), and by a grant of Chonnam National University Hospital Biomedical Research Institute (BCRI20062). The funding organizations had no role in the design or conduct of this research.

\section{Author Contributions}

Mi Sun Sung and Yong Sok Ji are sharing the first place within the authors. They both designed, recruited participants, collected, managed, analyzed and interpreted the data, and prepared the manuscript. Hyun Sik Moon and Hwan Heo collected and analyzed the data. Sang Woo Park designed and directed this study and prepared and reviewed the manuscript. All authors discussed the results and commented on and reviewed the manuscript. 


\section{References}

1 Watson PG, Young RD. Scleral structure, organisation and disease: a review. Exp Eye Res. 2004 Mar;78(3):609-23.

2 Bryant MR, McDonnell PJ. Optical feedback controlled scleral remodeling as a mechanism for myopic eye growth. J Theor Biol. 1998 Aug;193(4):613-22.

3 McBrien NA, Lawlor P, Gentle A. Scleral remodeling during the development of and recovery from axial myopia in the tree shrew. Invest Ophthalmol Vis Sci. 2000 Nov;41(12): 3713-9.

4 McBrien NA, Cornell LM, Gentle A. Structural and ultrastructural changes to the sclera in a mammalian model of high myopia. Invest Ophthalmol Vis Sci. 2001 Sep;42(10):217987.

5 Vurgese S, Panda-Jonas S, Jonas JB. Scleral thickness in human eyes. PLoS One. 2012; 7(1):e29692.

6 Norman RE, Flanagan JG, Rausch SM, Sigal IA, Tertinegg I, Eilaghi A, et al. Dimensions of the human sclera: thickness measurement and regional changes with axial length. Exp Eye Res. 2010 Feb;90(2):277-84.

7 Moring AG, Baker JR, Norton TT. Modulation of glycosaminoglycan levels in tree shrew sclera during lens-induced myopia development and recovery. Invest Ophthalmol Vis Sci. 2007 Jul;48(7):2947-56.

8 Deng J, Jin J, Lv M, Jiang W, Sun S, Yao C, et al. Distribution of scleral thickness and associated factors in 810 Chinese children and adolescents: a swept-source optical coherence tomography study. Acta Ophthalmol. 2019 May;97(3):e410-8.

9 Shinohara K, Yokoi T, Uramoto K, Takahashi H, Onishi Y, Horie S, et al. Posterior staphylomas and scleral curvature in highly myopic children and adolescents investigated by ultra-widefield optical coherence tomography. PLoS One. 2019 Jun;14(6):e0218107.

10 Ren R, Wang N, Li B, Li L, Gao F, Xu X, et al. Lamina cribrosa and peripapillary sclera histomorphometry in normal and advanced glaucomatous Chinese eyes with various axial length. Invest Ophthalmol Vis Sci. 2009; 50(5):2175-84
11 Maruko I, Iida T, Sugano Y, Oyamada H, Akiba M, Sekiryu T. Morphologic analysis in pathologic myopia using high-penetration optical coherence tomography. Invest Ophthalmol Vis Sci. 2012 Jun;53(7):3834-8.

12 Lopilly Park HY, Lee NY, Choi JA, Park CK. Measurement of scleral thickness using swept-source optical coherence tomography in patients with open-angle glaucoma and myopia. Am J Ophthalmol. 2014 Apr;157(4): 876-84.

13 Hayashi M, Ito Y, Takahashi A, Kawano K, Terasaki H. Scleral thickness in highly myopic eyes measured by enhanced depth imaging optical coherence tomography. Eye. 2013 Mar;27(3):410-7.

14 Buckhurst HD, Gilmartin B, Cubbidge RP, Logan NS. Measurement of scleral thickness in humans using anterior segment optical coherent tomography. PLoS One. 2015 Jul; 10(7):e0132902.

15 Pekel G, Yağcı R, Acer S, Ongun GT, Çetin EN, Simavlı H. Comparison of corneal layers and anterior sclera in emmetropic and myopic eyes. Cornea. 2015 Jul;34(7):786-90.

16 Dhakal R, Vupparaboina KK, Verkicharla PK. Anterior sclera undergoes thinning with increasing degree of myopia. Invest Ophthalmol Vis Sci. 2020 Apr;61(4):6.6

17 Xu L, Wang Y, Li Y, Wang Y, Cui T, Li J, et al. Causes of blindness and visual impairment in urban and rural areas in Beijing: the Beijing Eye Study. Ophthalmology. 2006 Jul;113(7): 1134.e1-e11.

18 Xu L, Wang Y, Wang S, Wang Y, Jonas JB. High myopia and glaucoma susceptibility the Beijing Eye Study. Ophthalmology. 2007 Feb; 114(2):216-20.

19 Sung MS, Heo H, Piao H, Guo Y, Park SW. Parapapillary atrophy and changes in the optic nerve head and posterior pole in high myopia. Sci Rep. 2020 Mar;10(1):4607.

20 Piao H, Guo Y, Ha JY, Sung MS, Park SW. Association of macular thickness with parapapillary atrophy in myopic eyes. BMC Ophthalmol. 2020 Mar;20(1):93.

21 Sung MS, Heo MY, Heo H, Park SW. Bruch's membrane opening enlargement and its implication on the myopic optic nerve head. Sci Rep. 2019 Dec;9(1):19564.
22 Sung MS, Heo H, Park SW. Microstructure of parapapillary atrophy is associated with parapapillary microvasculature in myopic eyes. Am J Ophthalmol. 2018 Aug;192:157-68.

23 Bland JM, Altman DG. Statistical methods for assessing agreement between two methods of clinical measurement. Lancet. 1986 Feb; 1(8476):307-10.

24 Ebneter A, Häner NU, Zinkernagel MS. Metrics of the normal anterior sclera: imaging with optical coherence tomography. Graefes Arch Clin Exp Ophthalmol. 2015 Sep;253(9): 1575-80.

25 Read SA, Alonso-Caneiro D, Vincent SJ, Bremner A, Fothergill A, Ismail B, et al. Anterior eye tissue morphology: scleral and conjunctival thickness in children and young adults. Sci Rep. 2016 Sep;6:33796.

26 Read SA, Alonso-Caneiro D, Free KA, LabucSpoors E, Leigh JK, Quirk CJ, et al. Diurnal variation of anterior scleral and conjunctival thickness. Ophthalmic Physiol Opt. 2016 May;36(3):279-89.

27 Zhang Q, Xu L, Wei WB, Wang YX, Jonas JB. Size and shape of Bruch's membrane opening in relationship to axial length, gamma zone, and macular Bruch's membrane defects. Invest Ophthalmol Vis Sci. 2019 Jun;60(7): 2591-8.

28 Greene PR. Mechanical considerations in myopia: relative effects of accommodation, convergence, intraocular-pressure, and the extraocular muscle. Am J Optom Physiol Opt. 1980 Dec;57(12):902-14.

29 Wang X, Fisher LK, Milea D, Jonas JB, Girard MJ. Predictions of optic nerve traction forces and peripapillary tissue stresses following horizontal eye movements. Invest Ophthalmol Vis Sci. 2017 Apr;58(4):2044-53.

30 Drexler W, Findl O, Schmetterer L, Hitzenberger CK, Fercher AF. Eye elongation during accommodation in humans: differences between emmetropes and myopes. Invest Ophthalmol Vis Sci. 1998 Oct;39(11):2140-7.

31 Sevel D. The origins and insertions of the extraocular muscles: development, histologic features, and clinical significance. Trans Am Ophthalmol Soc. 1986;84:488-526. 